\title{
Ultralow-loss silicon ring resonators
}

\author{
Aleksandr Biberman, ${ }^{1}$ Michael J. Shaw, ${ }^{2}$ Erman Timurdogan, ${ }^{1}$ Jeremy B. Wright, ${ }^{2}$ and Michael R. Watts ${ }^{1, *}$ \\ ${ }^{1}$ Research Laboratory of Electronics, Massachusetts Institute of Technology, 77 Massachusetts Avenue, Cambridge, Massachusetts 02139, USA \\ ${ }^{2}$ Sandia National Laboratories, P.O. Box 5800, Albuquerque, New Mexico 87185, USA \\ ${ }^{*}$ Corresponding author: mwatts@mit.edu
}

Received July 24, 2012; revised September 7, 2012; accepted September 10, 2012; posted September 11, 2012 (Doc. ID 173236); published October 9, 2012

\begin{abstract}
We experimentally demonstrate silicon ring resonators with internal quality factors of $Q_{0}=2.2 \times 10^{7}$, corresponding to record $2.7 \mathrm{~dB} / \mathrm{m}$ propagation losses. Importantly, we show that these propagation losses are limited by bend loss, indicating that the propagation loss limit for silicon has not yet been reached. (c) 2012 Optical Society of America
\end{abstract} OCIS codes: $230.4000,230.5750,230.7370$.

Waveguide loss is a key parameter for many important microphotonic devices and systems, ranging from highquality filters to delay lines, high-fidelity sensor systems [1] , and systems addressing many key challenges in computing and communication architectures [2]. Additionally, silicon is quickly proving to be the waveguide material of choice for microphotonics, enabling active componentry, such as modulators, switches, and photodetectors [3-5]; passive components, such as microring resonators and polarization transparent devices; and compact polarization splitters and rotators [ $\underline{6}-\underline{8}]$, as well as nonlinear devices, such as comb generators, wavelength converters, and time lenses $[9,10]$. The limit to propagation losses within silicon is an ongoing research area, however, and is yet to be determined.

Recent research efforts in the near-infrared spectrum have yielded a wide array of waveguides with low propagation losses. Silica waveguides have achieved about $0.037 \mathrm{~dB} / \mathrm{m}$ [11], and silica wedge resonators have achieved quality factors of $Q=8.75 \times 10^{8}$ [12]. Very weakly confined modes in silicon nitride waveguides reveal that losses as low as $0.045 \mathrm{~dB} / \mathrm{m}$ are possible [13]. Phosphorous-doped silica-on-silicon waveguides have achieved $0.85 \mathrm{~dB} / \mathrm{m}$ [14]. Highly confined silicon nitride waveguides have achieved about $10 \mathrm{~dB} / \mathrm{m}[15,16]$. Strip, locally oxidized, and ridge silicon waveguides have achieved 80 [17], 21 [18], and about $2.6-20 \mathrm{~dB} / \mathrm{m}$ [19-22], respectively. Silicon microdisk resonators have been shown to have propagation losses as low as $10 \mathrm{~dB} / \mathrm{m}$, with quality factors of $Q=5 \times 10^{6}$, limited by absorption in surface states [21]. In this Letter, we demonstrate a silicon ring resonator with propagation loss measured to be a record $2.7 \mathrm{~dB} / \mathrm{m}$.

Here, we apply techniques of reflowing photoresist [21], and oxidation-based smoothing to a ridge waveguide $[15,17]$. The ridge waveguide substantially reduces sidewall interaction with the fundamental TE and TM modes [Fig. 1(c)]. This enables quality factors as high as $2.2 \times 10^{7}$, corresponding to record-low propagation losses of $2.7 \mathrm{~dB} / \mathrm{m}$ in silicon, a factor of five lower than in Borselli et al. [21]. Moreover, TE and TM resonance propagation losses are two orders of magnitude different because of low-aspect-ratio ridge waveguide. By comparing the measured TE and TM resonances to numerical results obtained by a full-vectorial cylindrical modesolver, we demonstrate that the quality factors are limited by the incomplete confinement of the ridge waveguide, and the resulting bend-induced radiation, rather than fabrication or material-induced loss. Thus, even lower propagation losses are possible.

A silicon ridge waveguide design was chosen to demonstrate the low-loss waveguides with the assumption

(a)

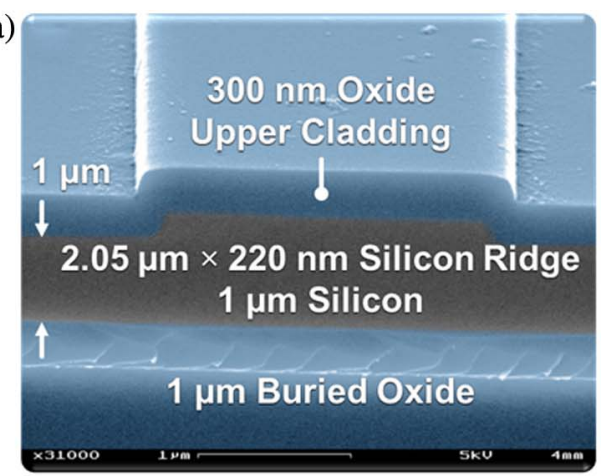

(b)
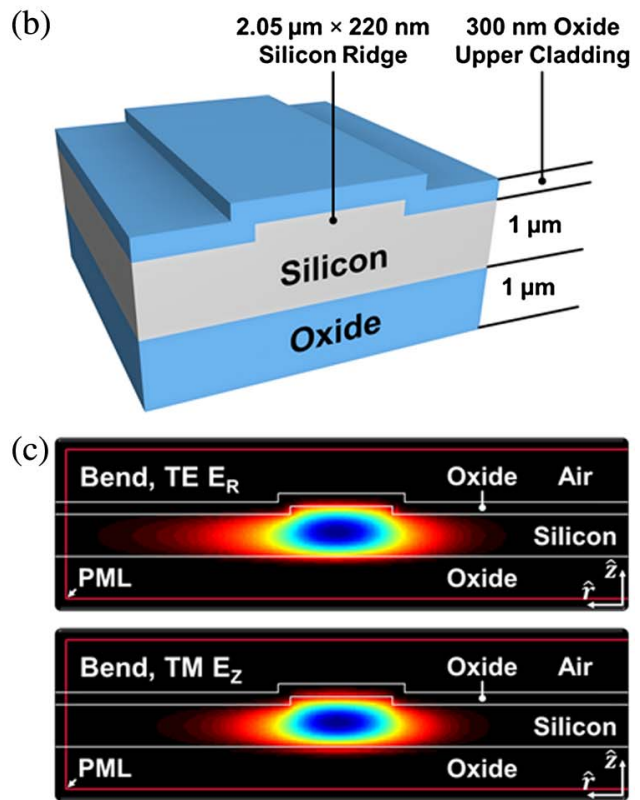

Fig. 1. (Color online) (a) Scanning-electron-microscope image of the silicon photonic waveguide overlaid with false coloring. (b) Representation of its layers and dimensions. (c) Modesolver simulations of the TE and TM modes at 1600 and $1554 \mathrm{~nm}$, respectively. The bend-loss-limited $Q$ factors from the modesolver simulations closely match the simulated results for both the TE and TM modes indicating that the measured $Q$ factors are limited by bend-loss-induced radiation. 
(a)

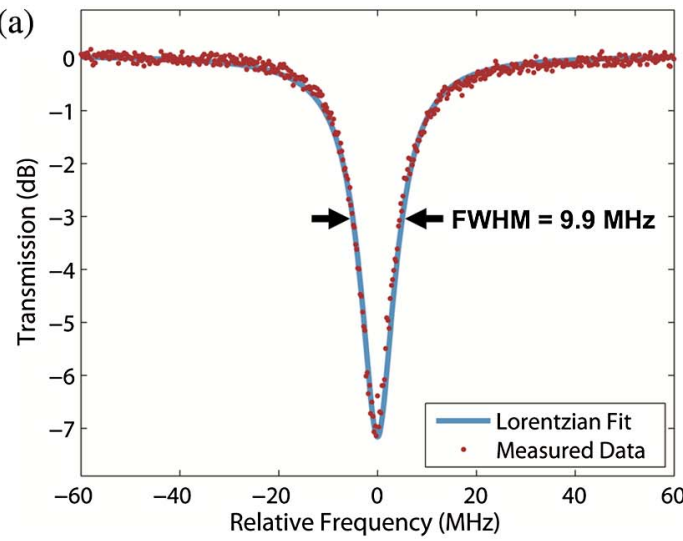

(b)

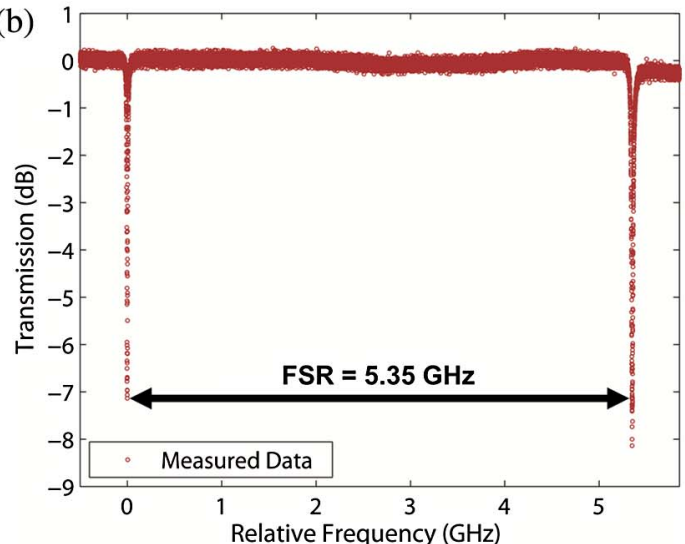

Fig. 2. (Color online) Experimentally measured TE spectral responses of the silicon photonic ring resonator. (a) Single passband, showing the FWHM, with a corresponding Lorentzian fit. (b) Two consecutive passbands, showing the free spectral range.

that reducing the sidewall interaction, with proper fabrication, could enable ultralow propagation losses. The waveguides were fabricated from a silicon-on-insulator (SOI) wafer, with a $3.26 \mu \mathrm{m}$ thick silicon layer and a $1 \mu \mathrm{m}$ thick oxide layer. The silicon layer was thinned to $1.33 \mu \mathrm{m}$ by oxidizing the wafer surface in a steam oxidation process followed by an oxide strip. A $200 \mathrm{~nm}$ thick ridge was patterned and etched to a silicon thickness of $1.13 \mu \mathrm{m}$ everywhere except the ridge; the total thickness of the ridge is $1.33 \mu \mathrm{m}$. Finally, a $300 \mathrm{~nm}$ thick thermal oxide layer was grown, consuming $0.13 \mu \mathrm{m}$ of silicon, and leaving behind a $200 \mathrm{~nm}$ thick ridge waveguide on a $1 \mu \mathrm{m}$ thick silicon slab. While patterning the ridge, a reflowed photoresist strategy was utilized [21], in which the photoresist was patterned and heated for a very short time. The top oxidation process and reflowed photoresist reduce or eliminate sidewall asperities created from the ridge etching process. A scanning-electron-microscope (SEM) image of the final waveguide is shown in Figure 1(a). The resulting ridge was measured to be $2.05 \mu \mathrm{m}$ wide and $220 \mathrm{~nm}$ tall on a $1 \mu \mathrm{m}$ slab [Fig. 1(b)].

The device in our experiments includes the aforementioned silicon photonic waveguide coupled to a ring resonator with a radius of $2.45 \mathrm{~mm}$. The gap between the waveguide and the ring resonator is $2.25 \mu \mathrm{m}$. Because the $\mathrm{TE}$ resonances of these ring resonators are so narrow, on the order of $10 \mathrm{MHz}$, accurate measurements of their
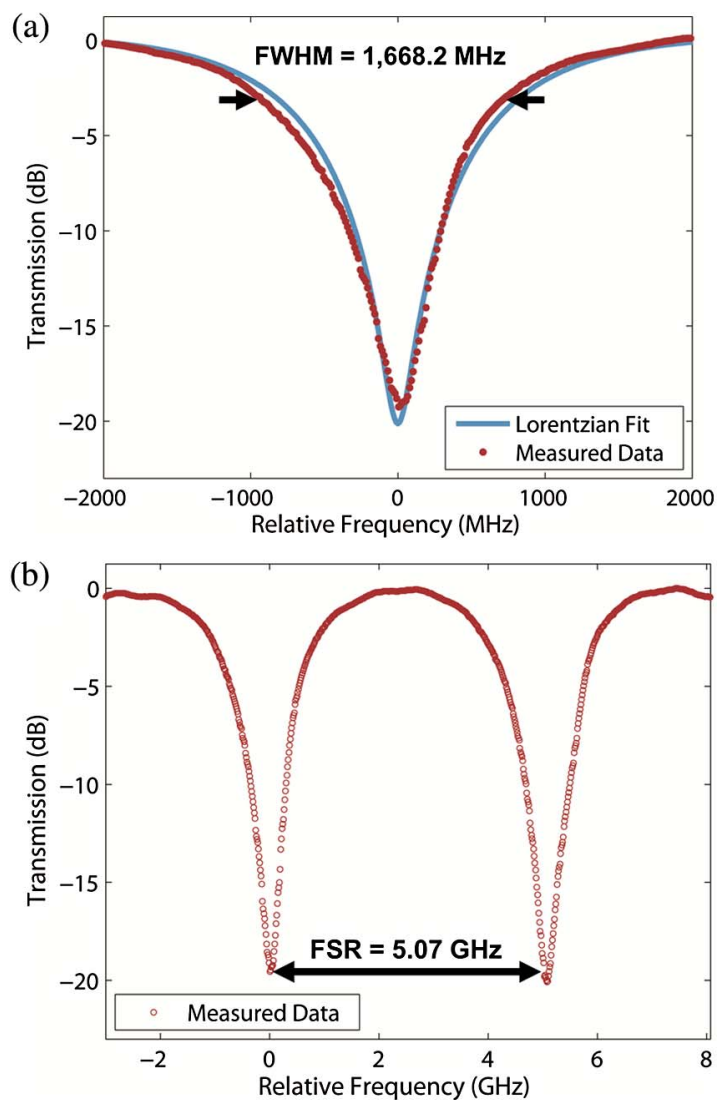

Fig. 3. (Color online) Experimentally measured TM spectral responses of the silicon photonic ring resonator. (a) Single passband, showing the FWHM, with a corresponding Lorentzian fit. (b) Two consecutive passbands, showing the free spectral range.

response is required for careful instrumentation. To measure the TE resonances, we continually swept a tunable laser source with a $100 \mathrm{kHz}$ linewidth over a $7 \mathrm{GHz}$ range, to span two consecutive resonances-that is, one free spectral range (FSR). The total fiber-to-fiber insertion loss was about $30 \mathrm{~dB}$ for the silicon photonic chip; there was about $-25 \mathrm{dBm}$ average optical power ingressing into the silicon ring resonator. As we swept the tunable laser source across the resonances, we obtained the spectrally correlated transmission in the time domain on an oscilloscope. Correlating the temporal duration between consecutive resonances to the FSR of the ring, separately measured using the tunable laser, we obtained calibrated curves for the TE spectral responses of the ring resonator, shown in Figs. 2(a) and 2(b). For the $\mathrm{TE}$ resonance at $1600 \mathrm{~nm}$, we measure a resonance width of $9.9 \mathrm{MHz}$. Fitting the data to a transfer matrix model of the Lorentzian resonance, we obtain an internal quality factor of $Q_{0}=2.2 \times 10^{7}$, and from a Taylor series expansion of the complex propagation constant $\left(\beta_{c}=\beta_{r}-j \alpha\right)$, we have $\alpha=\omega /\left(2 Q_{0} v_{g}\right)$, resulting in $\alpha_{\mathrm{TE}}=3.1 \times$ $10^{-3} \mathrm{~cm}^{-1}$ for the imaginary component of the complex propagation constant. This corresponds to a propagation loss of $2.7 \mathrm{~dB} / \mathrm{m}$, a record for silicon resonators. Additionally, we measure the spectral response of the TM mode centered at $1554 \mathrm{~nm}$, shown in Figs. 3(a) and 3(b). Here, we find the internal quality factor to be $\overline{Q_{0}}=1.4 \times 10^{5}$ from the fit and calculate $\alpha_{\mathrm{TM}}=0.5 \mathrm{~cm}^{-1}$. 
Inserting the dimensions of the ridge waveguide obtained from profilometry and SEM data, with refractive indices of 3.48 for silicon and 1.445 for silicon dioxide, we obtained the TE and TM fundamental modes and complex propagation constant of the structure using a full-vectorial cylindrical modesolver and perfectly matched layers. These modes are shown in Fig. 1(c). For the TE mode at $1600 \mathrm{~nm}$, we obtain $Q_{0}=2.2298 \times 10^{7}$ and $\alpha_{\mathrm{TE}}=3.1034 \times 10^{-3} \mathrm{~cm}^{-1}$. For the TM mode, we obtain $Q_{0}=1.3246 \times 10^{5}$ and $\alpha_{\mathrm{TM}}=0.5408 \mathrm{~cm}^{-1}$. The quality factors found from the simulation are consistent with our measured data. Therefore, our ring losses are limited by the radiation losses inherent in the realized structure and are not due to fabrication or other material limitations, such as free-carrier absorption, surface-state absorption and scattering, two-photon absorption, and defects. Thus, even higher quality factors for more compact devices are achievable by shrinking the ridge height or widening the ridge width.

We experimentally demonstrate a new record for ultralow-loss silicon ring resonators of $2.7 \mathrm{~dB} / \mathrm{m}$, achieving ring resonators with internal quality factors of $2.2 \times 10^{7}$, a factor of five better than what previously has been shown for resonators [21]. We confirm our results by simulating these structures using a full-vectorial cylindrical modesolver, showing that the major limitation in the quality factor in our ring resonators arises from the limited confinement factor of the ridge waveguide. With greater modal confinement, which can be readily achieved, we expect to achieve even lower propagation loss for even more compact resonators. The impact of such low loss in silicon waveguides and ring resonators will no doubt extend from communications and sensing applications, to delay lines and nonlinear optical effects.

Sandia National Laboratories is a multiprogram laboratory managed and operated by Sandia Corporation, a wholly owned subsidiary of Lockheed Martin Corporation, for the U.S. Department of Energy's National Nuclear Security Administration under contract DEAC04-94AL85000.

\section{References}

1. M. R. Watts, M. J. Shaw, and G. N. Nielson, Nat. Photonics 1, 632 (2007).
2. A. Biberman and K. Bergman, Rep. Prog. Phys. 75, 046402 (2012).

3. Q. Xu, B. Schmidt, S. Pradhan, and M. Lipson, Nature 435, 325 (2005).

4. M. R. Watts, W. A. Zortman, D. C. Trotter, R. W. Young, and A. L. Lentine, Opt. Express 19, 21989 (2011).

5. D. Ahn, C. Hong, J. Liu, W. Giziewicz, M. Beals, L. C. Kimerling, J. Michel, J. Chen, and F. X. Kärtner, Opt. Express 15, 3916 (2007).

6. M. Popovíc, T. Barwicz, M. R. Watts, P. T. Rakich, L. Socci, E. P. Ippen, F. X. Kärtner, and H. I. Smith, Opt. Lett. 31, 2571 (2006).

7. T. Barwicz, M. R. Watts, M. A. Popovic, P. T. Rakich, L. Socci, F. X. Kärtner, E. P. Ippen, and H. I. Smith, Nat. Photonics 1, 57 (2007).

8. D. Dai and J. E. Bowers, Opt. Express 19, 10940 (2011).

9. A. R. Johnson, Y. Okawachi, J. S. Levy, J. Cardenas, K. Saha, M. Lipson, and A. L. Gaeta, Opt. Lett. 37, 875 (2012).

10. W. Mathlouthi, H. Rong, and M. Paniccia, Opt. Express 16, 16735 (2008).

11. H. Lee, T. Chen, J. Li, O. Painter, and K. J. Vahala, Nat. Commun. 3, 867 (2012).

12. H. Lee, T. Chen, J. Li, K. Y. Yang, S. Jeon, O. Painter, and K. J. Vahala, Nat. Photonics 6, 369 (2012).

13. J. F. Bauters, M. J. R. Heck, D. D. John, J. S. Barton, C. M. Bruinink, A. Leinse, R. G. Heideman, D. J. Blumenthal, and J. E. Bowers, Opt. Express 19, 24090 (2011).

14. R. Adar, M. Serbin, and V. Mizrahi, J. Lightwave Technol. 12, 1369 (1994).

15. M. J. Shaw, J. Guo, G. A. Vawter, S. Habermehl, and C. T. Sullivan, Proc. SPIE 5720, 109 (2005).

16. A. Gondarenko, J. S. Levy, and M. Lipson, Opt. Express 17, 11366 (2009).

17. K. Lee, D. Lim, L. C. Kimerling, J. Shin, and F. Cerrina, Opt. Lett. 26, 1888 (2001).

18. F. Y. Gardes, G. T. Reed, A. P. Knights, G. Mashanovich, P. E. Jessop, L. Rowe, S. McFaul, D. Bruce, and N. G. Tarr, Proc. SPIE 6898, 68980R (2008).

19. P. Dong, W. Qian, S. Liao, H. Liang, C. Kung, N. Feng, R. Shafiiha, J. Fong, D. Feng, A. Krishnamoorthy, and M. Asghari, Opt. Express 18, 14474 (2010).

20. W. Bogaerts and S. K. Selvaraja, IEEE Photonics J 3, 422 (2011).

21. M. Borselli, T. J. Johnson, and O. Painter, Opt. Express 13, 1515 (2005).

22. G. Li, J. Yao, H. Thacker, A. Mekis, X. Zheng, I. Shubin, Y. Luo, J.-H. Lee, K. Raj, J. E. Cunningham, and A. V. Krishnamoorthy, Opt. Express 20, 12035 (2012). 